\author{
Sońa SOPÚCHOVÁ \\ Faculty of Law \\ Comenius University in Bratislava \\ sona.sopuchova@flaw.uniba.sk
}

\title{
ARTIFICIAL INTELIGENCE AND ITS USE IN THE PROCESS OF PROVIDING HEALTH CARE
}

\begin{abstract}
In the article, the author discusses the issue of electronic health care with a focus on telemedicine. The first part of the paper provides an overview and explanation of the basic related concepts, which are electronic healthcare and e-health, telemedicine and health care. In this part, the author also summarizes the relevant legislation. The author also presents the performance of telemedicine in the conditions of the Slovak Republic. Above all, the author asks which legal institutes are affected by another way of providing health care, which is distance medicine? The author gradually analyzes selected issues, namely instruction and informed consent, making audio or video-audio recording, recording telemedicine performance in medical documentation and payment for such performance. The conclusion of the article is devoted to summarizing and pointing out the threats and challenges of telemedicine, which the author considers the use of private communication platforms, the related violation of cyber security and the use of artificial intelligence. Key words: electronic healthcare, e-health, telemedicine, remote medicine, remote health care provision.
\end{abstract}

\section{INTRODUCTION}

In recent years, society has undergone many significant changes that permeate various areas of social life. The subject of changes are new ways of exercising rights, using services and also communication, when these activities lead to the massive introduction of information and communication technologies (hereinafter also referred to as "ICT"). These include a computer, mobile phone, 
tablet, TV, credit card and much more. In these cases, those are technical devices that allow information to be searched, retrieved, stored, transmitted and shared, thus greatly facilitating and streamlining various human processes and activities. In connection with the intensive expansion of information and communication technologies, it can be stated that we are becoming part of a society that is gaining the name of information ${ }^{1}$ and, given the speed of ongoing changes, we can talk about an information revolution. ${ }^{2}$ The Slovak Republic, like other member states of the European Union, has long been working to build an information society, the emergence and global dimension of which represents a logical outcome of the operation of information and communication technologies in all spheres of life. A characteristic feature of the information society, ie a society based on the use of ICT, is the continuous electronisation ${ }^{3}$ and creation of new departments with the electronic term, such as electronic public administration (e-Government), electronic justice (e-Justice), electronic banking (e-Banking) or electronic health care (e-Health) etc. It is interesting to note that these terms, which come from the English language, are often not translated into Slovak (or in other languages) and thus become part of the newspeak of modern society.

At present, the very discussed topic is the electronic health care-the so-called e-Health, which brings modern technologies into the exercise of important social law-the right to health protection. ${ }^{4}$ One of the sub-areas of e-Health is telemedicine, sometimes referred to as distance medicine. In addition to it, we also recognize e-Health services within e-Health, some of which have been processed in the Slovak Republic at the legislative level and in practice have found application in the form of legal institutes, such as electronic health book, e-prescription, e-examination and others. Technologies are moving forward very

1 The information society is defined as a society based on the penetration of information and communication technologies, information and knowledge into all areas of social life to such an extent that it fundamentally changes social relations and processes.

2 Ministry Of Education Of The Slovak Republic in cooperation with the Slovak Informatics Society and the Institute of Informatics and Statistics. Company informatization policy in the Slovak Republic. p. 2.

3 A process in which the emphasis is on the introduction and use of electronic information and communication technologies.

4 The provision of article 40 of the Constitutional Act no. 460/1992 Coll. The Constitution of the Slovak Republic. 
dynamically, bringing new possibilities and, given that many of them cannot be used without legal entrenchment, legislation should keep pace with them.

The aim of this article is to approach the issue of telemedicine in practice. We will focus on the provision of health care using information and communication technologies, issues of informing patients, recording doctors, recording telemedicine services, reimbursement by health insurance companies, and in the end we will touch on the practical challenges and threats of this modern form of health care provision.

\section{TheORETICAL BACKGROUND}

\section{BASIC CONCEPTS}

\section{E-HEALTH}

The term e-Health was first mentioned in 1999 at the 7th International congress of telemedicine and distance care in London, to express the stage of development of health sciences, especially health and medical informatics and telemedicine (the subject of interest is electronic health records, health informatics) and e-learning, computer networks for medical research and data transmission, health information system for patient ordering. ${ }^{5}$ As mentioned above, the term e-Health is often used without translation into Slovak, but in various forums we may come across the term e-Health or electronic healthcare. For this reason, these terms can be used as synonyms, and we will use them in other parts of the article, depending on the context. In 2003, the Conference of ministers of health of the member states of the European Union and other European countries also officially defined the term e-Health, as the use of modern information and communication technologies to meet the needs of citizens, patients, health professionals, healthcare providers and creators of health policy. The World Health Organization (WHO) also sees the importance of e-Health for public health and for health as a whole. Therefore, in 2005, it adopted Resolution WHA58.2.28 e-Health, which sets out its e-Health

\footnotetext{
5 Streda, l., Panýrek, P. eHealth a telemedicine, p. 16.
} 
strategy. It calls on member states to plan for the use of e-Health at national level. In this document, the WHO defines e-Health as an effective and safe way to use information and communication technologies to promote health and health-related activities; it includes the provision of health care, public health monitoring, but also medical literature and education, the knowledge base and scientific research. ${ }^{6}$ Electronic healthcare thus represents a comprehensive system of support for the provision of health care implemented through information and communication technologies. The vision of the e-Health project is to provide the right information at the right time, in the right place and in the right form at all stages and processes of citizens' health care.

\section{TELEMEDICINE}

Part of e-Health is telemedicine, which we refer to the provision of health care at a distance, and for this reason, telemedicine is also referred to as distance medicine. ${ }^{7}$ From the etymological point of view, the name telemedicine comes from the Greek and Latin languages. The Greek prefix tele - can be translated into a distance and the Latin term medeor means to heal. ${ }^{8}$ As was the case with e-Health, there are several definitions for telemedicine, whether in the European Union or the WHO.

The European Union has provided a definition of telemedicine in terms of the provision of healthcare services through the use of ICT in a situation where the healthcare professional and the patient (or two healthcare professionals) are not in the same place. The prerequisite is the secure transmission of medical data and information in the form of text, sound, images or in any other form necessary for the prevention, diagnosis, treatment and follow-up of patients. ${ }^{9}$ The World Health Organization defines telemedicine as health activities, services and systems operated remotely through information and

6 WHO. Resolution WHA58.28 eHealth, p. 121.

7 The word distance (medicine) is derived from the English word "distance", which means "distance".

8 Streda, L., Panýrek, P. eHealth a telemedicine, p. 15.

9 European Commission. Communication from the Commission to the European Parliament, the Council, the European Economic and Social Committee and the Committee of the Regions on telemedicine for the benefit of patients, healthcare systems and society, p. 3. 
communication technologies to support global health, prevention and health care, as well as education, health management and health research. ${ }^{10}$

Telemedicine thus represents a combination of medical informatics and telecommunications enabling remote data transmission, consulting activities and remote provision of medical and related services. While the beginnings of e-Health do not have a long history and we date them to recent decades, because the development of electronic health care is mainly related to the development of computer communication, the beginnings of telemedicine can be traced back. This term was previously used for remote medicine, even before the period of electronic society. ${ }^{11}$ The name telemedicine has stabilized in terminology mainly at the instigation of the WHO. Other definitions can be found in the United States of America within their American Telemedicine Association (ATA).

While e-Health as such is a significant part of multi-country health policy and includes a variety of services (including those that do not directly provide healthcare), telemedicine has remained focused on professional medical and therapeutic applications and its main purpose is to provide clinical support, overcome geographical barriers by connecting doctors and patients in different places through ICT in order to have a positive impact on health. Telemedicine communication is divided into verbal, visual and data. ${ }^{12}$ More precisely, data transmission is also used for telemedicine verbal and visual communication. However, this transmission is not immediate and takes place remotely as synchronous communication or asynchronous communication. While synchronous communication takes place at the same time, users respond with a delay in asynchronous communication.

We also include electronic healthcare services, which are related to the provision of healthcare through information and communication technologies, in the framework of electronic healthcare and telemedicine. These are, for example, an electronic medical record, an electronic patient summary, an electronic prescription of a medicine (so-called e-prescription),

10 WHO. Telemedicine: opportunities and developments in Member States: report on the second global survey on eHealth 2009, s. 9

11 Streda, L., Panýrek, P. eHealth a telemedicine, p. 15.

12 Streda, L., Panýrek, P. eHealth a telemedicine, p. 17. 
an electronic examination (so-called e-examination) or an electronic order (so-called e-order).

\section{LEGISLATION}

There is no doubt that the introduction of common e-Health rules is necessary to improve the patient's communication with the doctor, which can take place via (for example) the Internet. ${ }^{13}$ Decades ago, the countries of the European Union were called upon to draw up long-term strategic plans for the development and implementation of e-Health, and by signing the Lisbon Strategy in 2000, they committed themselves to genuinely creating programs for e-Health and telemedicine in particular. They stipulated that each state must strive to use ICT in healthcare as much as possible, regardless of the country in which the patient or information is located. The European Union's most recent strategic act is the 2012 document entitled eHealth Action Plan 2012-2020-Innovative Healthcare for the 21st Century, in which the European Commission commented on a number of important issues, such as the importance of telemedicine and its potential benefits, interoperability of e-Health information systems and protection of personal data.

Following the European steps, the Slovak Republic is adopting both strategic documents and legal regulations ensuring the accessibility of electronic healthcare services and the possibility of practicing distance medicine. The process of electronic health care in Slovakia is supported by several strategic documents, especially the Addendum to the National Reform Program of the Slovak Republic for 2006-2008, the Addendum to the National Reform Program of the Slovak Republic for 2008-2010, the Concept of Healthcare Informatisation for 2007-2010, the material Strategic Objectives eHealth from 2008, the new strategy of the eHealth Implementation Program of January 2011, which consists of several projects, and finally the Program Statement of the Government of the Slovak Republic for 2020-2024, in which the government undertakes to support the introduction of innovative modern technologies, such as such as telemedicine and the expansion of e-Health services. ${ }^{14}$

13 Streda, L., Panýrek, P. eHealth a telemedicine, p. 37.

14 The Government Of The Slovak Republic. Program Statement of the Government of the Slovak Republic for the Period 2020-2024, p. 37 and 40. 
In addition to strategic documents, which are not legally binding and their main task is to define visions and tools to meet those visions, legislation is extremely important for the implementation and use of telemedicine, especially laws and by-laws of ministries or other bodies. The legislation on the provision of health care represents an important and necessary set of rules that ensures the regulation of the right to health protection in relation to each person. Legislation governing the provision of healthcare, including telemedicine, includes the following legislation:

a. Act no. 576/2004 Coll. on health care, services related to the provision of health care and on amendments to certain acts (hereinafter also referred to as the "Health Care Act"),

b. Act no. 578/2004 Coll. on health care providers, health care workers, professional organizations in health care and on the amendment of certain laws,

c. Act no. 581/2004 Coll. on health insurance companies, supervision of health care and on amendments to certain acts (hereinafter also referred to as the "Act on Health Insurance Companies"),

d. Act No. 153/2013 Coll. on the national health information system and on amendments to certain acts (hereinafter also referred to as the "NZIS Act").

For the possibilities of telemedicine, it is important to include in particular the Health Care Act, which regulates the provision of health care both in general and in some levels of distance medicine. Clarification of the question of which specific areas of telemedicine are regulated and can therefore be implemented will be the subject of the next part of the article. From the point of view of electronic healthcare in general, the latter NZIS Act is important, which defines key terms and defines important processes such as the national health information system, the procedure for accessing data from the national health information system, national administrative registers, electronic health book or patient summary.

Several pieces of legislation, including those in the medical field, have been amended several times during their period of validity, but numerous modifications have been recorded in the past 2020 due to the pandemic caused by the COVID-19 viral disease. While in the past period telemedicine in Slovakia was mentioned only marginally, nowadays it is an increasingly discussed topic, 
which is gradually reflected in the legislation and therefore finds application in practical life. A key amendment concerning telemedicine is the amendment to the Health Care Act. It is contained in Act no. 125/2020 Coll. amending Act no. 578/2004 Coll. on health care providers, health care workers, professional organizations in health care and on the amendment of certain laws as amended and which amends certain laws and introduces electronic doctor's consultations into practice. This is an element of telemedicine, which we will discuss in more detail in the next chapter of this article. However, we point out here that the possibility of using this form of telemedicine is currently legally limited to the duration of the crisis situation. The question, of course, is why this is the case and how this situation will develop after the end of the pandemic.

The aim of the legislative amendments is, among other things, to harmonize the Slovak Republic with the standardization process of e-Health in the European Union, to ensure mutual compatibility and interoperability, as well as to ensure the fulfillment of e-Health's vision in implementing the related e-Government program.

\section{Performance of Telemedicine in THE SLOVAK REPUBLIC}

Telemedicine is an area of e-Health, which is the provision of remote healthcare using ICT. Even before we focus on telemedicine, it is appropriate to clarify healthcare as such.

Healthcare has its legal definition in the provision of $\$ 2$ par. 1 of the Health Care Act, where it is defined as a set of work activities performed by health professionals, including the provision of medicines, medical devices and dietetic food to prolong the life of a natural person, improve their quality of life and healthy development of future generations; health care includes prevention, dispensary treatment, diagnosis, treatment, biomedical research, nursing care and midwifery. ${ }^{15}$ Under current legislation, healthcare and related services can be provided either by a healthcare provider and /

15 The provision of $\$ 2$ par. 1 of the Health Care Act. 
or by a healthcare professional, through health services as the basic units of healthcare provision. Furthermore, it should be mentioned in this context that healthcare providers are obliged to provide healthcare correctly. This means that healthcare professionals should perform all medical procedures to correctly identify the disease with timely and effective treatment to heal or improve the patient, taking into account current medical science and in accordance with standard prevention procedures, standard diagnostic procedures and standard therapeutic procedures taking into account the individual condition of the patient. ${ }^{16}$ In the Slovak Republic, health care is provided in several forms. They are: outpatient care, institutional care, pharmacy care and nursing care in social assistance facilities. ${ }^{17}$

Telemedicine is intended mainly for communication between a doctor and a patient and for collecting data from the patient via the internet, mobile devices or a telephone. The use of telemedicine in individual medical disciplines has certain common elements. Remote clinical care helps remove barriers and improves access to health services. It is both beneficial in emergency and intensive care, as well as in the care of chronic patients.

Regardless of the medical field in which telemedicine is used, its basic areas of activity can be divided into four categories:

a. transfer of information,

b. remote monitoring,

c. distance therapy,

d. telemedicine training. ${ }^{18}$

All the mentioned areas of telemedicine can be used in the outpatient form of health care provision. There are already telemedicine technologies that are successfully used in practice. These include monitoring of blood pressure and heart rate in heart disease, blood sugar levels in diabetes, spirometry in lung disease and asthma, the results of which can then be consulted with a doctor, either directly in person or remotely. ${ }^{19}$

16 The provision of $\$ 4$ par. 3 of the Health Care Act.

17 The provision of $\$ 7$ par. 1 of the Health Care Act.

18 Streda, L., Panýrek, P. eHealth a telemedicine, p. 68.

19 For remote monitoring, measurements can currently be provided through the following devices: ECG monitor, ECG holter Faros, glucometer, oximeter, infrared thermometer, continuous thermometer, pressure gauge, digital scale, pulse oximeter, smart bracelet, spirometer and coaguchek. 
Real-time communication between sender and recipient is not required when transferring information. Most often it is about sending pictures, e.g. radiodiagnostic or histopathological. Remote monitoring, on the other hand, requires real-time communication, which allows the doctor to respond immediately to the patient's initiative, for example in a videoconference. However, there is also "store and forward" technology ${ }^{20}$, which is the ability to transfer data from the patient to the doctor with a subsequent response from the doctor. It is common to monitor parameters such as blood pressure, weight or blood glucose. Long-distance therapy works on the principle of the geographical distance between the doctor and the patient. Its subject can be diagnostic and therapeutic procedures mediated by technology. Telemedicine education is closely linked to data sharing between doctors. Video conferencing, where doctors can get acquainted with methods applied in other workplaces, can be considered a benefit here. ${ }^{21}$

After a theoretical analysis of telemedicine methods, we will further focus on finding out the legislative possibilities of telemedicine in the conditions of the Slovak Republic with subsequent inclusion in a specific theoretical area.

At present, telemedicine can be performed in the Slovak Republic on the basis of the provision of $\$ 49 \mathrm{k}$ par. 1 of the Health Care Act, according to which: During a crisis situation, a general practitioner or a doctor with specialization is entitled, after verifying the identity of the person and the insurance relationship with his / her relevant health insurance company, to provide consultation. The provision of consultation via electronic communications must be recorded by the doctor in the person's medical records. ${ }^{22}$

In this case, the legislator had in mind outpatient care, because experience has shown that the provision of consultations by telephone or e-mail is common, but these have not yet been supported by law (e.g. verification of results by telephone, health consultation, etc.). The condition for the provision of health care in this form is the verification of the identity and insurance relationship of the insured with his / her respective health insurance company by the health care provider. Reimbursement of this medical performance is the subject of an agreement between the health insurance company and the health care provider in the health care contract, where

\footnotetext{
20 It means "save and pass on."

21 Streda, L., Panýrek, P. eHealth a telemedicine, p. 69-70.

22 The provision of $\$ 49 \mathrm{k}$ par. 1 of the Health Care Act.
} 
in such a case it is obligatory to agree on the process of verifying the identity and insurance relationship of the insured directly by the health insurance company. ${ }^{23}$ Further information on the means of consultation by electronic communication and their content is not stated in the law, but we can assume that, in the sense of telemedicine theory defined above, this may involve remote monitoring (with immediate or later doctor response) or remote therapy (subject to which may be diagnostic or therapeutic performance). We consider it important to mention the limitation of this (currently) only telemedicine option, and only during the crisis situation, which means that after its completion, consultations via electronic communication will not be possible without further amendment of the legislation. This is due to the fact that this provision was adopted by the National Council of the Slovak Republic only as a temporary one. In the next part of the article we will focus on selected issues related to the performance of telemedicine, which is available in Slovakia at the time.

\section{INFORMED PATIENT CONSENT AND INSTRUCTION}

Informed consent is a legal institute which in the conditions of the Slovak Republic is regulated in the Health Care Act as a demonstrable consent to the provision of health care preceded by an instruction and it is also a demonstrable consent to the provision of health care preceded by a refusal of instruction, unless otherwise provided by law. ${ }^{24} \mathrm{As}$ is clear from the legal text, informed consent must be preceded by instruction, which is also regulated by law, as follows: The healthcare professional is obliged to inform about the purpose, nature, consequences and risks of healthcare provision, about the options of proposed procedures and risks of refusal provision of health care, unless otherwise provided by this act. ${ }^{25}$ The attending healthcare professional is obliged to provide instruction clearly, considerately, without coercion, with the possibility and sufficient time to freely decide for informed consent and in proportion to the intellectual and voluntary maturity and state of health of the person to be instructed. The attending healthcare

23 Explanatory memorandum to the proposal Act no. 125/2020 Coll. amending Act no. 578/2004 Coll. on health care providers, health care workers, professional organizations in health care and on the amendment of certain laws. Special part, p. 7.

24 The provision of $\$ 6$ par. 4 of the Health Care Act.

25 The provision of $\$ 6$ par. 1 of the Health Care Act. 
professional is also obliged to record in the relevant electronic medical record in the electronic medical record information on whether the person has been instructed, whether the person has refused, whether the person has given informed consent or refused it, resp. whether she later recalled him. ${ }^{26}$

Informed consent of the patient, preceded by an instruction, is perhaps the most important prerequisite for the provision of health care. It is an important part of the modern understanding of health care and significantly activates the patient in the treatment process, while increasing his legal and medical awareness. In the case of an electronic consultation, the question arises as to whether informed consent and instruction can also be given at a distance. The answer can be found directly in the legislation on informed consent. The wording of the law states that informed consent must be provable. However, in theory and in practice, this does not necessarily mean its expression in written or even paper form. ${ }^{27}$ Thus, informed consent can also be given in another verifiable manner, for example orally in front of a witness, which may be a nurse in an outpatient clinic.

In order to be able to comprehensively answer the question of whether informed consent and instruction can also be given at a distance in the practice of telemedicine, we must return to the concept of health care, because informed consent agrees to its provision. However, is there an electronic consultation within the meaning of provision of $\$ 49 \mathrm{k}$ of the Health Care Act? So is informed consent and instruction necessary in this case? We are of the opinion that yes, because part of health care is prevention, diagnosis and treatment, which can be carried out at a distance as part of remote monitoring and remote therapy, ie without the patient's presence in the doctor's office. In this case, informed consent and instruction is required. If a doctor provides telemedicine as part of his or her outpatient clinic, they may proceed on the basis of the above, namely the possibility of expressing the patient's oral informed consent in front of a witness. Another possibility is to send a signed informed consent from the patient via, for example, an email, which can be considered as an electronic transfer of

26 The provision of $₫ 6$ par. 2 of the Health Care Act.

27 In some specific cases provided by law (eg sterilization), written consent is required. However, on the other hand, there are situations provided by law where healthcare can be provided without the patient's consent, for example in the case of urgent healthcare if informed consent cannot be obtained in time or in the case of institutional care if the person is spreading a communicable disease. which seriously endangers its surroundings. 
information-a category of telemedicine. In this context, we recall that an indication of whether an instruction has been given and whether informed consent has been given or the instruction has been refused must be recorded by means of an electronic medical record in the electronic medical record.

\section{MAKING VIDEO AND AUDIO RECORDINGS OF THE DOCTOR AND THE MEDICAL PROCEDURE}

Making a video and audio recording of a doctor and his medical performance or patient is not subject to the Health Care Act. In this case, we mean primarily the recording of a doctor in the provision of health care, on the basis of which a video and audio recording will be created. Because the doctor provides health care to a particular person, it is likely that the recording will include the patient's portrait or expression. However, this is not the rule, because, for example, a patient's likeness or manifestation may not be recorded during surgery. This issue is regulated in Act no. 40/1964 Coll. the Civil code, as amended (hereinafter also referred to as the "Civil Code"), which in provision of $\$ 11$ regulates the protection of personality, according to which every natural person has the right to protection of his personality, especially life and health, civil honour and human dignity, as well as privacy, his name and expressions of a personal nature. ${ }^{28}$ Furthermore, documents of a personal nature, portraits, images and video and audio recordings relating to a natural person or his expressions of a personal nature may be made or used only with his permission. ${ }^{29}$ The law therefore determines the conditions for the production and use of video and audio recordings of natural persons. The basic principle for making and using expressions of a personal nature is that they may be made and subsequently used only with the consent of the person to whom they relate. The consent must cover both acts, because if an unauthorized copying has taken place, in practice in particular of portraits, images or video and audio recordings, consent to their use cannot replace consent to their creation. This means that if a doctor or patient is interested in making a video and audio recording of a medical procedure, in our case,

\footnotetext{
28 Provision of $\$ 11$ of the Civil Code.

29 Provision of $\$ 12$ par. 1 of the Civil Code.
} 
for example, telemedicine, it is necessary to have the consent of the persons who will be the subject of the recording. The same will apply if person is interested in using such a record. The law does not specify how consent must be expressed, which implies that it can be both written and oral consent. In practice, the consent may seem such that the making of a video and audio recording is announced in advance by its creator and the presence of a person in a given situation means his consent. For example, a doctor alerts a patient at the beginning of a video call that the call is being recorded, and if the patient agrees, remains present, and if disagrees, but should agree that the doctor cannot coerce and make the telemedicine procedure tolerate recording.

In this context, however, we point out that in the event that the personal data of a natural person is processed in the course of video and audio recording, it is necessary to apply personal data protection legislation, according to which the personal data controller must have a legal basis for personal data processing which may be the performance of the contract, the law, the legitimate interest or consent of the person concerned and others. ${ }^{30}$ In the case of making a video and audio recording of a telemedicine recording, a legal basis will be required in this case-the consent of the person concerned. ${ }^{31}$

For unambiguous proof of a person's consent, both in accordance with the Civil Code and in accordance with Act no. 18/2018 Coll. on personal data protection and on amendments to certain acts (hereinafter also referred to as the "Personal Data Protection Act"), the reproduction or expression of which will be the subject of a visual and audio recording, its written form is strongly recommended. As part of the performance of telemedicine, it can be purchased in the form of an email message containing a digitized document including the given consent.

30 The provision of $\$ 13$ par. 1 of Act no. 18/2018 Coll. on the protection of personal data and on the amendment of certain laws.

31 With the consent of the affected person in accordance with Act no. 18/2018 Coll. on the protection of personal data and on the amendment of certain laws is any serious and freely given, specific, informed and unambiguous expression of the will of the data subject in the form of a statement or unambiguous confirmatory act by which the data subject consents to the processing of his personal data. 


\section{RECORDING OF TELEMEDICINE PERFORMANCE IN MEDICAL RECORDS}

Medical records are among the key institutes of health care delivery. It is a set of data on a person's state of health, health care and services related to the provision of health care to that person. ${ }^{32}$ There are several exceptions to this rule, and thus to cases where medical records are kept in writing..$^{33}$ The act also defines the content of the entry in the medical documentation and thus in accordance with the provisions of $\$ 20$ par. 3 of the Health Care Act, it should include:

a. date and time of registration,

b. the method of instruction, the content of the instruction, refusal of instruction, informed consent, refusal of informed consent and withdrawal of informed consent,

c. the date and time of the provision of health care, if different from the date and time of registration,

d. the scope of provided health care and services related to the provision of health care, including prescribed or administered human medicines, medical devices and dietetic foods,

e. the results of other examinations, if they are part of the provided healthcare for which registration is performed,

f. identification of the attending healthcare professional,

g. the identification of the person to whom the healthcare has been provided.

The obligation to register in medical records has also affected the provision of telemedicine in our country, because the transitional provision by which the legislator introduced the possibility to provide consultations by electronic communication explicitly establishes this obligation of a doctor. The truth is that the provision of $\$ 20$ par. 3 of the Health Care Act regulates situations where a person who is provided with health care is present in an outpatient clinic. At that time, of course, the legislator did not foresee that healthcare would be provided at a distance. When we go through the individual

32 The provision of $\$ 2$ par. 6 of the Health Care Act.

33 Let us make a brief remark here. Enrollment in the electronic medical record is also made in writing. Therefore, we believe that the term "written" should be replaced by "paper" in this context. 
information to be included in the registration, we do not find any mention of electronic consultation, but it comes closest to being included under letter d), which indicates the scope of provision of health care and related services. We believe that it should be added precisely because this provision does not contain the registration of an electronic consultation, the provision itself is explicitly contained in the provision of $\$ 49 \mathrm{k}$ of the Health Care Act regulating the distance type of consultations.

Related to the issue is the question of whether a written record or an entry in an electronic medical book is required. In this case, the performance of telemedicine is on the same level as other methods of providing health care, e.g. the provision of $\$ 20$ of the Health Care Act and the resulting exceptions apply to the form of registration, if they can be used. The mere provision of an electronic consultation does not justify the need to register in medical records.

\section{REIMBURSEMENT OF TELEMEDICINE SERVICES}

According to the Explanatory Memorandum to the draft of the Health Care Act, the payment of electronic consultation as a form of telemedicine should be part of an agreement between a health insurance company and a health care provider in a contract on the provision of health care. It must also include an agreement on the process of verifying the identity and insurance relationship of the insured directly by the health insurance company, e.g. the insurance company and the healthcare provider must agree in advance on how the provider will verify the identity and existence of the long-distance patient insurance relationship ${ }^{34}$. The existence of the health insurance of the person to whom the telemedicine service is to be provided can be considered a basic legal condition for the reimbursement of telemedicine services by the health insurance company. After the inclusion of an electronic consultation in the Health Care Act, each health insurance company operating in the Slovak Republic has issued guidelines on how to correctly report the performance

34 Explanatory memorandum to the proposal Act no. 125/2020 Coll. amending Act no. 578/2004 Coll. on health care providers, health care workers, professional organizations in health care and on the amendment of certain laws. Special part, p. 7. 
of telemedicine. ${ }^{35}$ The Ministry of Health of the Slovak Republic also issues various methodological guidelines and standard procedures. One of them is the Standard Procedure for the Provision of Healthcare in the General Outpatient Clinic for Adults during the COVID-19 pandemic, within which Annex no. 5-Principles of distance consultation. This document explains how the doctor-patient consultation should take place at a distance, but in this case it is mainly a consultation on COVID-19.

There are currently four types of telemedicine coverage that are covered by public health insurance:

a. Performance $1 \mathrm{~b}-$ Consultation by e-mail or telephone

b. Performance 11a-Consultation through enhanced electronic communication in the online environment (web application, video call)

c. Exercise 70-Re-issuance of prescriptions and / or vouchers at the patient's request by e-mail, SMS or telephone

d. Exercise 1c-Crisis psychotherapeutic intervention or individual psychotherapy or psychodiagnostic interview with the patient via telephone or video call

In addition to the legal condition for the reimbursement of telemedicine services, which is health insurance, there are other conditions set by health insurance companies in their guidelines. In particular, it must be a matter of providing relevant medical advice to the insured person or his legal representative with regard to his state of health and while maintaining the correctness of the provision of health care in accordance with the provisions of $₫ 4$ par. 3 of the Health Care Act. Furthermore, the above-mentioned telemedicine services cannot be combined with each other or with other services and the tools used for electronic communication must meet the basic security parameters from the point of view of IT and from the point of view of personal data protection. It follows from the above that if a patient is interested in using the possibility of electronic communication and consultation with his doctor during a crisis situation, he is not obliged to pay for these services as above-standard health

35 The General Health Insurance Company issued a public promise pursuant to the provisions of provision of $\$ 850$ et seq. of Civil Code, which publicly promised all providers of general and specialized outpatient care that the health care provided from 1 April 2020 until the revocation of the state of emergency declared in connection with the Covid-19 pandemic, resp. until the revocation of this promise, it shall pay under the specified conditions which form part of the public promise. 
care if he is a participant in public health insurance. In this context, we add that in the Slovak Republic there is a method of declaring and verifying the identity of persons in a virtual space in the form of an electronic ID card, which could also be used to verify identity during electronic consultation, if it takes place, for example in the form of email or other long distance communication.

\section{CONCLUSION-THREATS AND CHALLENGES OF TELEMEDICINE}

Electronic healthcare (e-Health) and its component-telemedicine are a relatively new issues in the conditions of the Slovak Republic, their implementation has already begun and patients can use several electronic services such as electronic prescription or electronic health book, but especially in the case of telemedicine we observe several legal limits.

For the threats of telemedicine, we identified the topic of personal data protection and the related use of private platforms for electronic communication between doctor and patient. Pursuant to the Health Care Act, the patient's consent is not required for the processing, provision and access to data from health documentation under the conditions laid down in a special regulation, which is the NZIS Act. ${ }^{36}$ This means that the healthcare provider is entitled to process data from medical records without obtaining the patient's consent, and therefore, in accordance with the Personal Data Protection Act, the legal basis for processing in this case is a special legal regulation. ${ }^{37}$ In this context, it is important to note that in the case of healthcare, a special type of personal data is processed, which the Personal Data Protection Act calls health data. These are expressly prohibited to process by the Personal Data Protection Act. However, as it is not possible not to process such data in the case of health care provision, the law provides for an exception where the prohibition of processing this category of personal data does not apply if the processing is necessary for preventive occupational medicine, health care and health care services. For the purpose of providing public health insurance, if

\footnotetext{
36 The provision of $\$ 18$ par. 2 of the Health Care Act.

37 The provision of $\$ 13$ par. 1, letter c) of the Personal Data Protection Act.
} 
these data are processed by a healthcare provider, a health insurance company, a person performing services related to the provision of health care or a person supervising health care and on his behalf a professionally qualified person who is bound by a duty of confidentiality, which the person learned during the performance of the activity, and the obligation to comply with the principles of professional ethics. ${ }^{38}$

Although the processing of personal data contained in medical records does not require the consent of the patient under certain conditions, the doctor has several obligations to fulfil in particular the obligation to provide information to anyone who processes personal data and also the obligation to comply with the permitted scope of processing personal data. However, in this context and in connection with the protection of personal data, a problematic point arises, namely electronic communication within telemedicine carried out via various modern platforms, such as Zoom, Teams, Messenger, Skype or even classic private mail. The legal basis here can be considered the regulation of the health care provider's obligations related to the provision and storage of medical records and the provision and disclosure of data from it, which must take place in accordance with the Health Care Act and special regulations (Personal Data Protection Act and General Regulation on personal data). The provision of medical records is the responsibility of the provider, who is obliged to store and protect it so that it is not damaged, lost, destroyed or misused. ${ }^{39}$ Furthermore, the provision and making available of data from medical records is regulated relatively strictly, especially in relation to the addressees and the methods of providing and making available. We distinguish between the provision and disclosure of data from medical records kept in writing at the provider, when the Health Care Act applies, and the provision and disclosure of data from the electronic health record, which are covered by the NZIS Act. Electronic sharing of digital data can be done through electronic healthcare information systems by healthcare professionals or through an electronic medical record by the patient. In both cases, however, what we have stated above applies that the healthcare provider is obliged to protect the medical documentation and to provide and

\footnotetext{
38 The provision of $\$ 16$ par. 2 of the Personal Data Protection Act.

39 The provision of $\$ 22$ par. 1 of the Health Care Act.
} 
make the data available only to entities subject to tax law. And they do not include a legal entity or a natural person operating the mentioned platforms used for electronic communication, which in case of sharing through their tool may have this data at their disposal. For this reason, each tool used for electronic communication between doctor and patient must meet the basic parameters of IT security and personal data protection, data transmission in this form of remote communication must be ensured in such a way that the content of communication must not be available to the tool operator. Such security can be achieved by encrypting communication at all times and not distributing it to other application servers, and last but not least, the patient registration tool must not require any additional data other than that necessary for the actual start of (for example) a video call. It is the responsibility of the telemedicine healthcare provider to comply with these rules. In the event that telemedicine in the form of electronic consultation finds its permanent legislative anchoring and practical application in the Slovak Republic, the state should set the mentioned parameters of IT security in the form of specific and uniform standards, so that they do not remain only in the form of recommendations.

Another potential threat may be a breach of cybersecurity, which means a breach of the confidentiality, integrity and availability of information in cyberspace. Information, in this case, for example, data from medical records provided in the context of telemedicine, may be affected by a cyberattack causing a cyber security incident in the form of information leakage, alteration or misuse. If the goal is electronic health information systems, there may also be harm to the life or health of individuals. Such situations can be illustrated by a real example of a ransomware called "Wannacry" paralyzing the British National Health Service to such an extent that patient data could not be accessed and almost 19,000 examinations and hundreds of surgeries had to be delayed. A similar type of misconduct may arise in relation to an individual, e. g. obtaining information in the context of telemedicine and the subsequent intimidation or blackmail of a healthcare professional. There is no doubt that the more electronic and digitized our society is, the more cyber security issues need to be addressed. 
The use of modern information and communication technologies in healthcare, on the other hand, also brings positive aspects and challenges. As an example, we mention artificial intelligence, which is gradually finding application in the field of healthcare. This technology can be applied to various aspects of health care, such as providing a system for analyzing medical information to identify sources of error and develop solutions using existing results and adding computer intelligence to medical devices and instruments.

This article was drafted with the support from a grant awarded by the Slovak Research and Development Agency No. APVV - 17 - 0403 Effects of Mutual Recognition of Electronic Identification Means on Electronic Services of Public Administration and is included in a research task. 


\section{REFERENCES}

Andraško, J. (2021)et al. Law of information and communication technologies 2. Bratislava: TINCT. p. 324, ISBN: 978-80-073837-2-5

Ministry Of Education Of The Slovak Republic. Company informatization policy in the Slovak Republic.

European Commission. (2008). Communication from the Commission to the European Parliament, the Council, the European Economic and Social Committee and the Committee of the Regions on telemedicine for the benefit of patients, healthcare systems and society.

National Audit Office. (2018). Investigation: WannaCry cyber attack and the NHS. Available at: https://www.nao.org.uk/wp-content/uploads/2017/10/InvestigationWannaCry-cyber-attack-and-the-NHS.pdf

Description. (2009).Electronic healthcare services. p. 206

Streda, L., Panýrek, P. (2011). eHealth and Telemedicine. Prague: High Tech Park. p. 159, ISBN 987-80-254-9508-7

Explanatory memorandum to the proposal Act no. 125/2020 Coll. amending Act no. 578/2004 Coll. on health care providers, health care workers, professional organizations in health care and on the amendment of certain laws. Special part

The Government Of The Slovak Republic. Program Statement of the Government of the Slovak Republic for the Period 2020 - 2024

\section{ACT}

Act no. 576/2004 Coll. on health care, services related to the provision of health care and on the amendment of certain laws

Act no. 18/2018 Coll. on the protection of personal data and on the amendment of certain laws

Act no. 40/1964 Coll. Civil Code

Constitutional Act no. 460/1992 Coll. The Constitution of the Slovak Republic

WHO. (2005). Resolution WHA58.28 eHealth.

WHO. (2009). Telemedicine: opportunities and developments in Member States: report on the second global survey on eHealth. 